\title{
Mixoma de características atípicas: un simulador en tiempos de COVID-19
}

\author{
Alberto Piserra López-Fernández de Heredia* \\ Jorge Perea Armijo* \\ Mónica Delgado Ortega** \\ Dolores Mesa Rubio**
}

Jesús Rodríguez Nieto*
Correspondencia

Alberto Piserra López-Fernández de Heredia alberto.piserra.lopez@gmail.com

\footnotetext{
* Servicio de Cardiología. Hospital Universitario Reina Sofía. Córdoba. España

** Servicio de Cardiología. Unidad de Imagen Cardíaca. Hospital Universitario Reina Sofía. Córdoba. España
}

\section{Recibido: $21 / 04 / 2020$ \\ Aceptado: 02/06/2020 \\ En línea: 31/07/2020}

Citar como: Piserra-López-Fernández de Heredia A, Perea-Armijo J, Rodríguez-Nieto J, Delgado-Ortega M, Mesa-Rubio D. Mixoma de características atípicas: un simulador en tiempos de COVID-19. Rev Ecocar Pract (RETIC). 2020 (Jul); 3 (2): 29-32. doi: 10.37615/retic.v3n2a9.

Cite this as: Piserra-López-Fernández de Heredia A, Perea-Armijo J, Rodríguez-Nieto J, Delgado-Ortega M, Mesa-Rubio D. Atypical myxoma features: A simulator in times of COVID-19. Rev Ecocar Pract (RETIC). 2020 (Jul); 3 (2): 29-32. doi: 10.37615/retic.v3n2a9.

\section{Palabras clave}

$\triangleright$ Mixoma auricular quístico

$\triangleright$ Mixoma auricular atípico

\section{RESUMEN}

Se presenta el caso de una mujer de 55 años con un mixoma en aurícula izquierda de morfología atípica. Fue ingresada por un cuadro de fiebre, tos y disnea de 1 mes de evolución asociado con la aparición de infiltrados alveolares en la radiografía de tórax. La ecocardiografía reveló la existencia de una masa en aurícula izquierda dependiente del septo interauricular, heterogénea y con un gran componente quístico, que prolapsaba a través de la válvula mitral causando obstrucción significativa. Sus características morfológicas ecocardiográficas hicieron pensar en un mixoma o un sarcoma como dos posibles diagnósticos etiológicos. Fue intervenida de forma urgente y la biopsia intraoperatoria confirmó la existencia de un mixoma auricular.

ABSTRACT
We present a case of a 55-year-old woman with an atypical myxoma in the left atrium. She was admitted for
one-month history of fever, cough and dyspnea associated with radiological alveolar infiltrates in the chest X-ray.
Echocardiography revealed the existence of a heterogeneous mass in the left atrium dependent on the interatrial
septum with a large cystic content prolapsing through mitral valve and causing significant obstruction. Its echo-
cardiographic morphological characteristics suggested myxoma or sarcoma as two possible etiological diagno-
ses. Urgently surgical intervention was practiced and the intraoperative biopsy confirmed the existence of atrial
myxoma.

\section{ABSTRACT}

$>$ Cystic atrial myxoma $\triangleright$ Atypical atrial myxoma

\section{Presentación del caso}

Una paciente sin antecedentes personales de interés acudió en enero de 2020 por un cuadro clínico de astenia, disnea con los esfuerzos, tos y febrícula de 1 mes de evolución, que hizo sospechar la presencia de una infección respiratoria. Se inició tratamiento antibiótico, pero la paciente no mejoró, con fiebre persistente, disnea en aumento y empeoramiento de los signos radiológicos (Figura 1).

En la analítica no se observó leucocitosis ni otras alteraciones significativas. La antigenuria para neumococo y Legionella, así como la microbiología para diferentes microorganismos resultaron negativas. Al presentarse la paciente 1 semana antes de la situación de alerta por pandemia de SARS-Cov-2 no se le realizó cribado de esta etiología.

Se solicitó una ecocardiografía transtorácica en la que se objetivó una función biventricular conservada y una tumoración en aurícula izquierda, dependiente del septo interauricular (Figura 2), que interfería en el funcionamiento valvu- lar mitral causando obstrucción al flujo con un gradiente medio de $7 \mathrm{mmHg}$ (Figura 3). Ante estos hallazgos se realizó una ecocardiografía transesofágica para valorar de forma más precisa las características de la masa. El estudio mostró una masa dependiente de septo interauricular que ocupaba la mayor parte de aurícula izquierda, con una composición heterogénea con una parte sólida y una gran zona quística que prolapsaba por la válvula mitral causando estenosis e insuficiencia (Vídeo 1 a Vídeo 5). Los dos posibles diagnósticos de presunción fueron mixoma atípico y sarcoma cardíaco.

Se decidió operar a la paciente. En la intervención se aprecia una masa intracardíaca redondeada de bordes bien definidos y aspecto gelatinoso con base de implantación en torno a fosa oval con aspecto macroscópico de mixoma en aurícula izquierda (Figura 4), diagnóstico que se confirmó histológicamente con una biopsia intraoperatoria. Se realizó una resección de la masa y de los bordes circundantes, con ampliación de la fosa oval y reconstrucción del septo interauricular con un parche de pericardio autólogo. Presentó buena evolución en el postoperatorio. El estudio anatomopatológico confirmó el diagnóstico de mixoma y fue dada de alta a la semana tras la intervención. 


\section{Estudio por imagen}

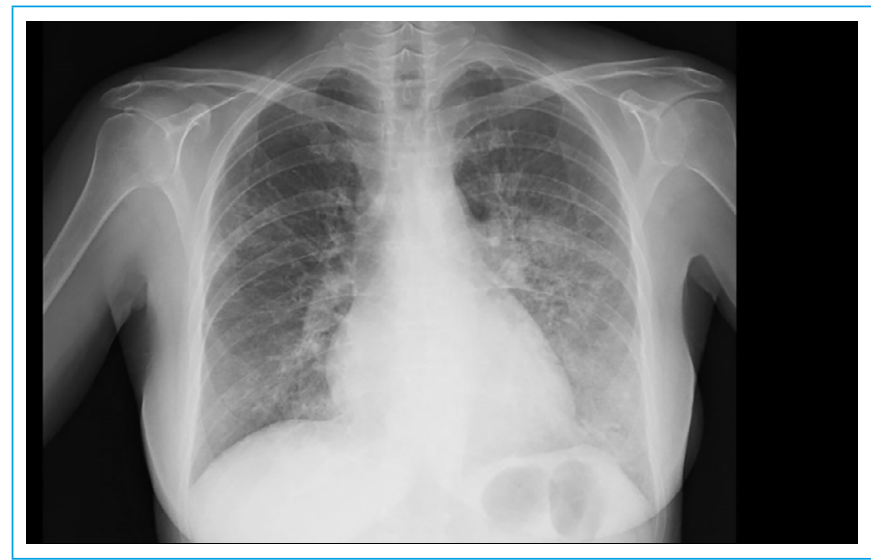

Figura 1. Radiografía PA de tórax. Hilios congestivos con patrón de redistribución vascular e infiltrado bilateral en bases sin cardiomegalia ni derrame pleural

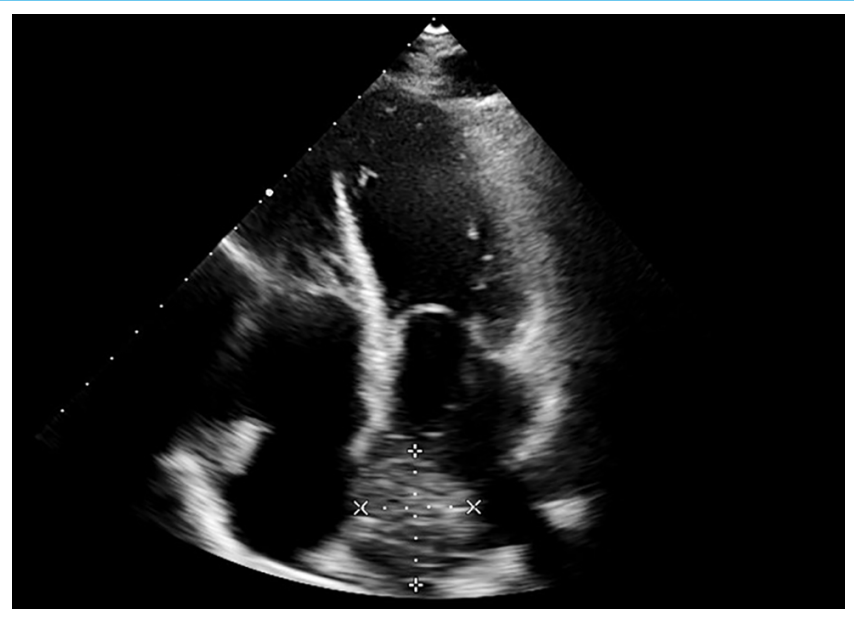

Figura 2. Ecocardiografía bidimensional transtorácica en plano apical de cuatro cámaras. Se observa una masa heterogénea en la aurícula izquierda de $3 \times 3 \mathrm{~cm}$, dependiente del septo interauricular

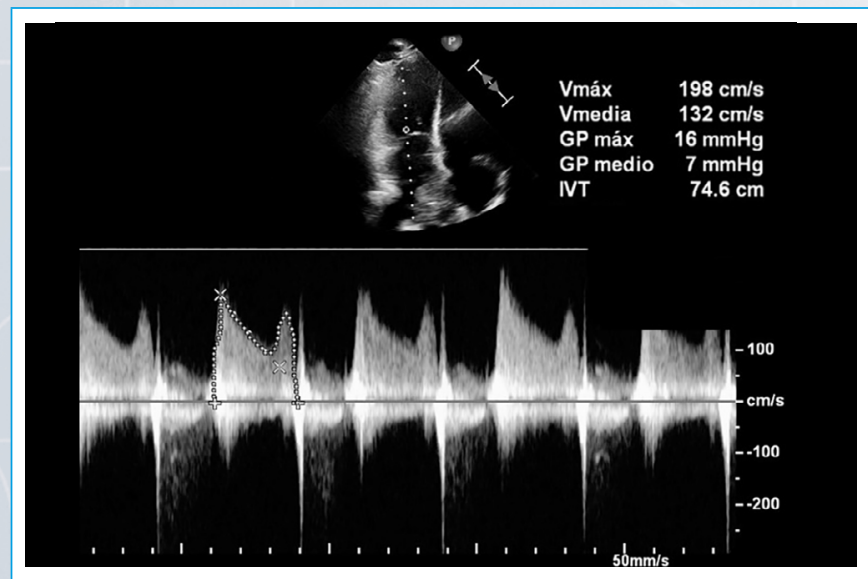

Figura 3. Ecocardiografía bidimensional transtorácica en plano apical de cuatro cámaras con Doppler continuo a través de la válvula mitral. Se aprecia aceleración del flujo con un gradiente transmitral medio de $7 \mathrm{mmHg}$

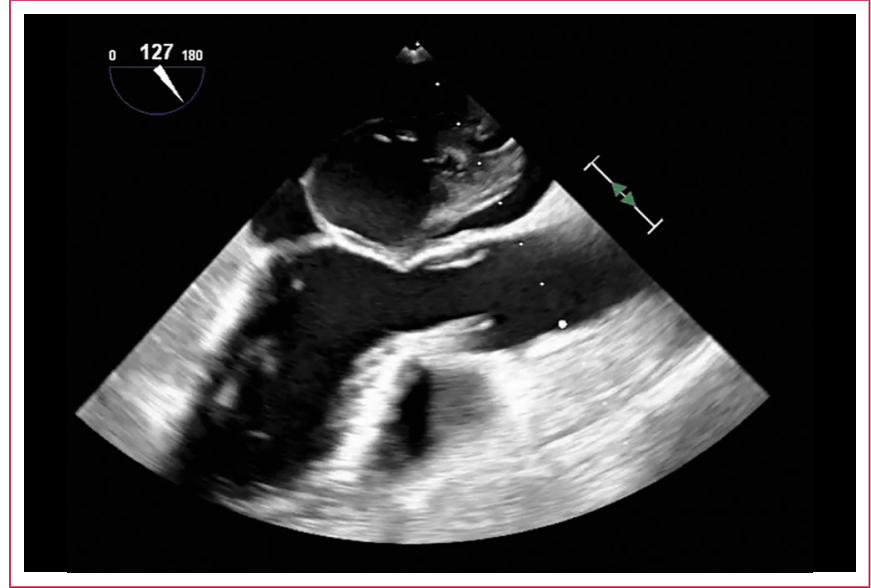

Vídeo 1. Ecocardiografía bidimensional transesofágica a nivel medioesofágico con ángulo de $120^{\circ}$. En el plano de tracto de salida del ventrículo izquierdo se observa una masa con dos componentes diferenciados. El primero de ellos se trata de una masa heterogénea con zonas anecoicas que sugieren necrosis y hemorragia, que ocupa la aurícula izquierda prácticamente en su totalidad. El extremo distal de esta masa corresponde a una imagen quística, encapsulada con interior anecoico, que protruye en diástole a través de la válvula mitral. Se aprecia una válvula mitral morfológicamente normal

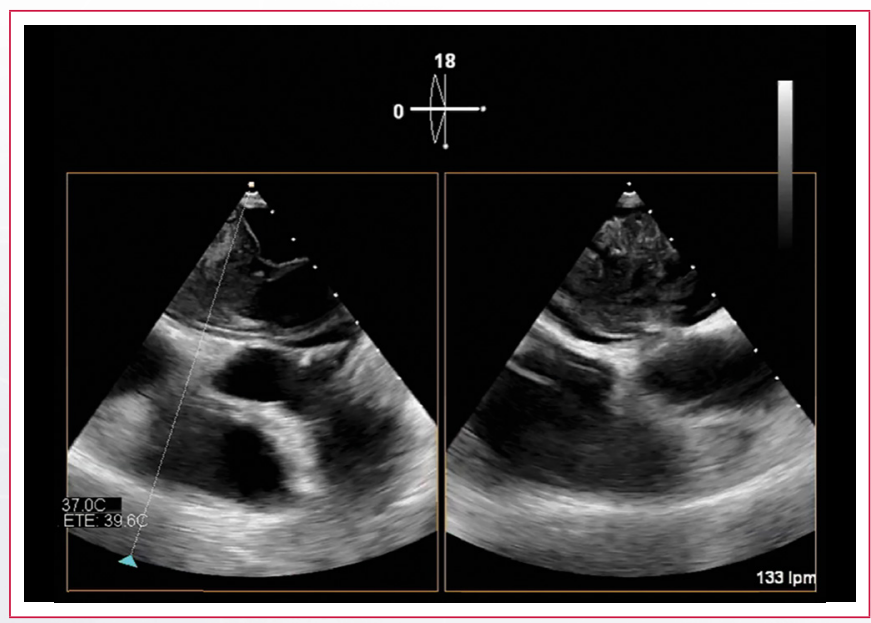

Vídeo 2. Ecocardiografía tridimensional transesofágica en visión X-plane. Se aprecia la masa en una región sólida en aurícula izquierda y otra en región quística atravesando el plano de la válvula mitral

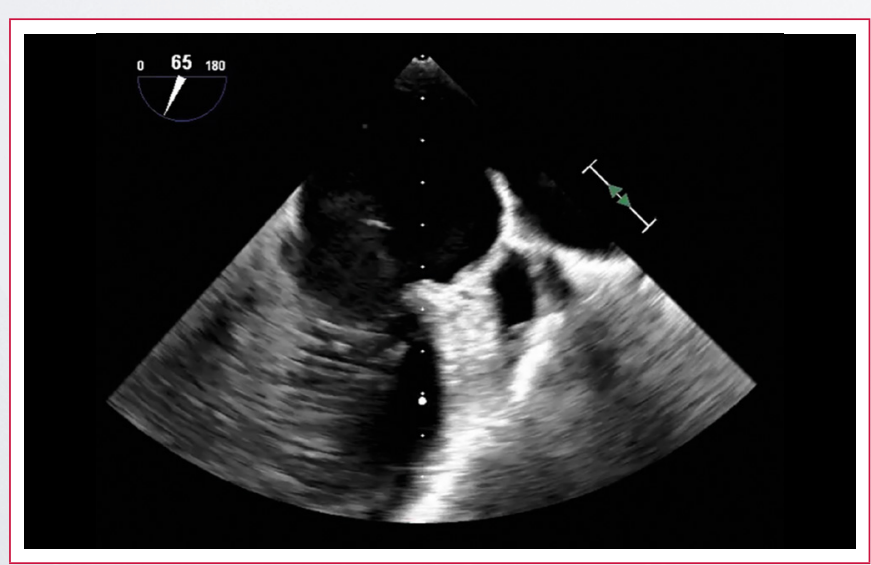

Vídeo 3. Ecocardiografía bidimensional transesofágica a nivel medioesofágico a $65^{\circ}$. Valoración en plano intercomisural de la porción quística de la masa atravesando la válvula mitral en diástole 


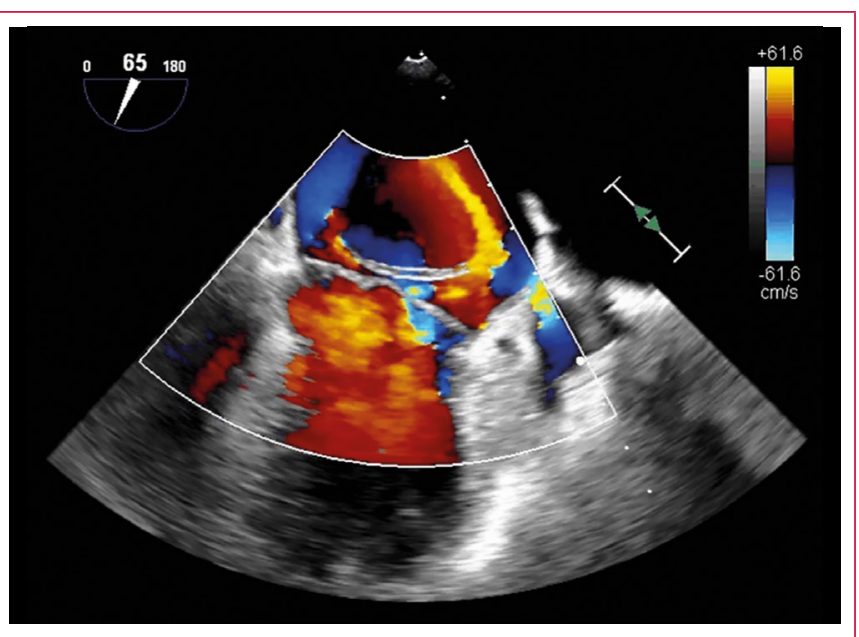

Vídeo 4. Ecocardiografía bidimensional transesofágica en plano medioesofágico a $65^{\circ}$ con Doppler color. Plano intercomisural de válvula mitral. Se aprecian dos jets de llenado turbulentos a través de válvula mitral, a ambos lados de la masa

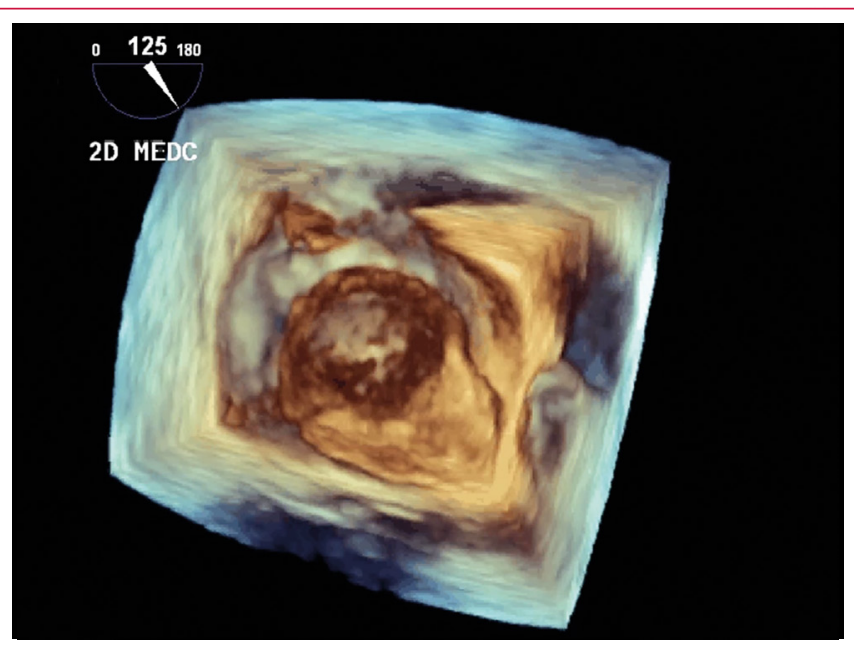

Vídeo 5. Ecocardiografía transesofágica tridimensional. Vista de la masa desde el techo de aurícula izquierda, con un componente sólido adherido al septo y quístico protruyendo a ventrículo izquierdo a través de válvula mitral

\section{Discusión}

Ante el diagnóstico de una masa cardíaca, hay que fijarse en varios aspectos durante la valoración ecocardiográfica. Su localización intracardíaca o extracardíaca, su lugar y modo de implante, su relación con las estructuras adyacentes, su forma y tamaño, y las consecuencias hemodinámicas que genera. Según estas características, se podrá establecer una sospecha acerca de su etiología benigna o maligna ${ }^{(1)}$

El mixoma es el tumor primario benigno cardíaco más frecuente, representa alrededor del 50\% de los casos. Puede aparecer de forma aislada o, menos frecuentemente, asociarse a formas familiares heredadas de forma autosómica dominante. Estos últimos casos habitualmente se presentan a edades más tempranas, de forma múltiple y recurren tras la cirugía más frecuentemente ${ }^{(1)}$. Clínicamente se manifiesta de forma variable, siendo la tríada clásica obstrucción, embolización y síntomas constitucionales inespecíficos ${ }^{(1)}$

La sintomatología de la paciente (fiebre, tos, disnea) y los infiltrados radiológicos, en el contexto actual hubiera sido altamente sospechoso de infección por COVID-19 y habría sido necesaria la extracción de muestras microbiológicas para SARS-Cov- $2^{(2)}$. Al presentarse 1 semana antes del auge de casos COVID-19 en España, a pesar de ser catalogado de infección neumónica, no se realizó cribado de SARS-Cov-2 como etiología del cuadro. Se ha documentado un descenso en los principales síndromes cardiovasculares durante la pandemia de COVID-19, llevando a un retraso en el diagnóstico y en el tratamiento de patologías con mortalidad elevada y tiempo-dependientes ${ }^{(3)}$. Se cree que casos como el descrito deben servir para no perder la sospecha de etiología cardíaca en pacientes con cuadros clínicamente sugestivos o disnea de evolución tórpida, a pesar de la lógica alta sospecha inicial de neumonía vírica en el momento en el que nos encontramos.

Típicamente, los mixomas se localizan en la aurícula izquierda y se trata de un tumor bien definido, de morfología globular e irregular habitualmente con un pedículo estrecho que asienta en el septo interauricular, cercano a la fosa oval(1). Tienen un tamaño medio de $4-8 \mathrm{~cm}$, y su superficie puede ser lisa (mixomas polipoideos), tendiendo a provocar obstrucción al flujo, o rugosa (mixomas papilares), con mayor tendencia a la embolización. Se presentan como masas sólidas, pero en ocasiones pueden presentar zonas ecolucentes y quísticas, correspondiendo a zonas de necrosis y hemorragia, especialmente los mixomas polipoideos ${ }^{(1)}$. Si bien es cierto que se describe la posibilidad de
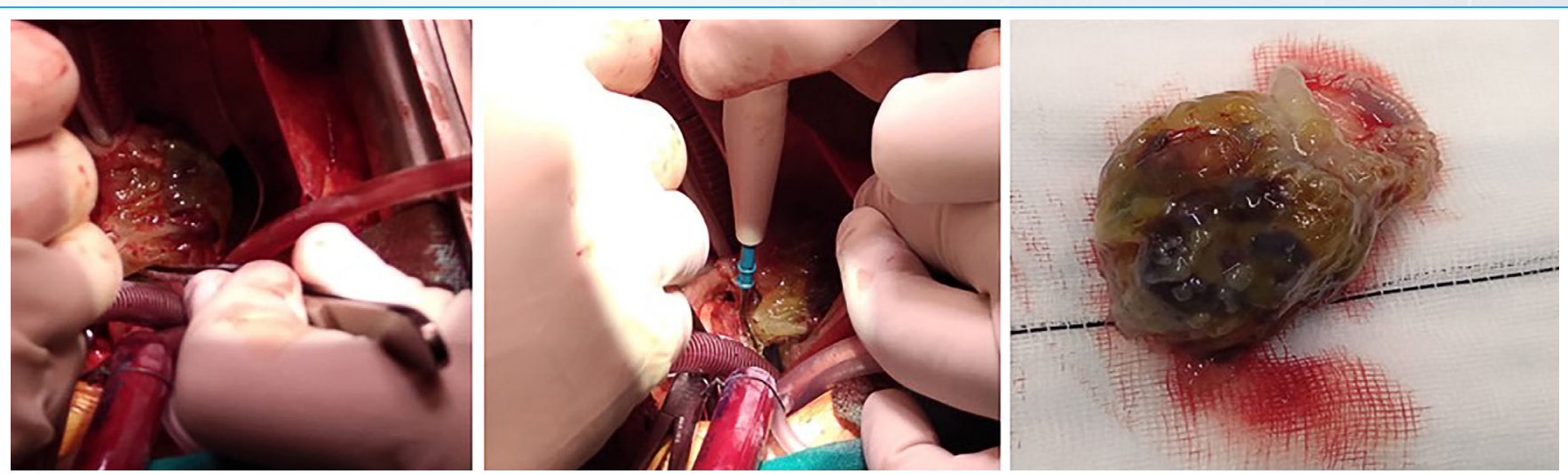

Figura 4. Imágenes intraoperatorias y de la pieza quirúrgica: masa pediculada anclada en el septo interauricular. La pieza presenta zonas de necrosis y hemorrágicas y una cápsula independiente a la derecha de la imagen que albergaba el contenido quístico observado en la ecocardiografía 
presentar zonas quísticas, son pocos los casos descritos en la literatura, siendo el mixoma quístico una variante atípica y poco frecuente ${ }^{(4)}$.

Atendiendo a la localización de este caso en la aurícula izquierda, otras posibilidades hubieran sido el lipoma o la presencia de trombos y, en menor frecuencia, las metástasis y el sarcoma ${ }^{(5)}$. Este último constituye el tumor primario maligno más frecuente y habitualmente se instaura en las cavidades derechas, especialmente en la aurícula derecha. Cuando se localiza en la aurícula izquierda, lo hace habitualmente en la pared posterior ${ }^{(1)}$. Suelen ser tumores sésiles, de ecogenicidad heterogénea, con áreas hipoecogénicas de necrosis, que podrían recordar a las características morfológicas de la masa tumoral de nuestra paciente.

Los lipomas son los segundos tumores cardíacos benignos en frecuencia y se presentan con más frecuencia en aurícula derecha, ventrículo izquierdo o pericardio, como masas habitualmente de pequeño tamaño, homogéneas y encapsuladas, con base de implantación amplia(1).

Los trombos cardíacos son masas homogéneas bien delimitadas, con algo más de ecogenicidad que el tejido circundante. La presencia de masas en zonas de estasis sanguíneo como la orejuela izquierda o áreas discinéticas del ventrículo izquierdo deben hacer sospecharlos. Podrían presentarse en la aurícula izquierda, por ejemplo, en pacientes con estenosis mitral y el hecho de no estar adherido a ninguna estructura cardíaca y su densidad más homogénea ayudarían a distinguirlos del mixoma ${ }^{(1)}$

Por las características quísticas de la masa, se podría plantear el diagnóstico diferencial con un quiste hidatídico cardíaco. Sin embargo, ni la localización era la típica (asientan en un 60\% de los casos en la pared del ventrículo izquierdo( ${ }^{(6)}$ ), ni las características clínicas acompañaban esta sospecha.

Este caso compartía características típicas de mixoma (masa localizada en au rícula izquierda, pediculada y dependiente del septo interauricular a nivel de la fosa oval), así como características atípicas (heterogenicidad y áreas quísticas sugerentes de necrosis, hemorragia y vascularización), que no permitían descartar la etiología de sarcoma cardíaco, por lo que tanto el mixoma auricular, como el sarcoma cardíaco izquierdo fueron las dos entidades planteadas como diagnóstico diferencial, con sus consecuentes diferencias en manejo intraoperatorio y pronóstico.

\section{Conclusión}

Las masas cardíacas son un problema clínico relativamente infrecuente. La clínica inespecífica que originan, junto con su baja incidencia, pueden hacer que el diagnóstico de sospecha se plantee tardíamente. La ecocardiografía transtorácica e idealmente la transesofágica constituyen la piedra angular del diagnóstico inicial y pueden orientar por las características de la masa y su localización hacia un tipo u otro de tumor, con diferente manejo y pronóstico. Sin embargo, esta clasificación no siempre es fácil y en ocasiones se combinan características de morfología y localización de diferentes masas cardíacas, por lo que resulta de vital importancia la asociación con la clínica y características del paciente.

A pesar del contexto epidemiológico actual de pandemia de COVID-19, no se debe olvidar la alta prevalencia de las enfermedades cardiovasculares y mantener esta sospecha en el diagnóstico diferencial de los pacientes.

\section{Ideas para recordar}

- El mixoma auricular es el tumor cardíaco benigno primario más frecuente y se presenta ecocardiográficamente de forma característica como una masa móvil, sólida y pediculada dependiente del septo interauricular.

- En ocasiones se pueden encontrar mixomas atípicos, heterogéneos o con contenido líquido encapsulado, como es el caso del mixoma quístico.

- Algunas patologías cardiovasculares de alta incidencia comparten características clínicas y radiológicas con la enfermedad COVID-19 y no se deben perder de vista en el diagnóstico diferencial, puesto que el manejo del paciente difiere por completo.

\section{Bibliografía}

1. Mingo Santos S, Moñivas Palomero V, Mitroi DC. Tumores y otras masas cardiacas. En: Cabrera. Ecocardiografía, 1. a ed. Madrid. Editorial Panamericana, $2011 ; 279-288$.

2. Ministerio de Sanidad. Procedimiento de actuación frente a casos de infección por el nuevo coronavirus (2019-nCoV). Centro de Coordinación de Alertas y Emergencias Sanitarias. Dirección General de Salud Pública. Versión del 11 de Abril de 2020.

3. Rodríguez-Leor O, Cid-Álvarez B, Ojeda S, et al. Impacto de la pandemia de COVID-19 sobre la actividad asistencial en cardiología intervencionista en España. REC Interv Cardiol 2020; 2: 82-89.

4. Lee KT, Lai WT, Yen HW, et al. Cystic left atrium myxoma. A rare case report. Kaohsiung J Med Sci 2001; 17: 579-581.

5. Dujardin KS, Click RL, Oh JK. The role of intraoperative transesophageal echocardiography in patients undergoing cardiac mass removal. Journal of the American Society of Echocardiography 2000; 13: 1080-1083.

6. Yaman ND, Sirlak M. Cardiac hydatid cyst. Review of recent literature. J Vet Med Res 2017; 4 (8): 1102. 